\title{
Functional near-infrared spectroscopy of prefrontal cortex during memory encoding and recall in elderly with type 2 diabetes mellitus
}

\author{
Fei Zhao, Machiko R. Tomita, Anirban Dutta
}

\begin{abstract}
Low-frequency Fahræus-Lindqvist-driven (not blood pressure-driven) oscillations in the small vessels are crucial because oscillations in small vessels support nutrient supply. Understanding of this is critical in type 2 diabetes mellitus (T2DM) to develop therapeutic measures to prevent Alzheimer's Disease Related Dementias: vascular factors contribute to cerebrovascular disease as well as mild cognitive impairment and dementia, which are predicted to affect 152 million people by 2050 (Alzheimer's Disease International London, UK, 2019). In this clinical study, we performed functional near-infrared spectroscopy (fNIRS) of the forehead to investigate the effect of the Mini-Cog with three-item recall test on the prefrontal cortex (PFC) activation and the relative oscillatory power in the $0.01-0.02-H z$ (Fahræus-Lindqvist effect) and $0.021-0.052 \mathrm{~Hz}$ (smooth muscle autonomic innervation) frequency bands in elderly (60 years and older) T2DM and age-matched controls. We found a significant (p<0.01) difference in the PFC activation between elderly subjects with T2DM and age-matched elderly controls. Moreover, power spectral density (PSD) analysis revealed a significantly lower relative power in $0.021-0.052 \mathrm{~Hz}$ (smooth muscle autonomic innervation) frequency band in elderly subjects with T2DM during the Mini-Cog three-item recall test. Furthermore, a drop in the oscillatory power in the 0.01-0.02$\mathrm{Hz}$ frequency band during Mini-Cog three-item recall test was found more pronounced in the elderly subjects with T2DM. Therefore, our study highlighted portable brain imaging to capture cerebrovascular reactivity to cognitive load that may provide a biomarker of cerebrovascular dysfunction in T2DM.

Clinical Relevance - Our study establishes forehead portable brain imaging for monitoring cerebrovascular function in T2DM under cognitive load.
\end{abstract}

\section{INTRODUCTION}

Global Burden of Disease Study of 369 diseases and injuries in 204 countries and territories (GBD 2019 Diseases and Injuries Collaborators) showed that diabetes significantly increases age-standardized disability-adjusted life years by $24.4 \%$ (from 18.5 to 29.7 years) [1]. Moreover, the U.S. burden of Alzheimer's Disease Related Dementias (ADRDs) is projected to double by 2060, according to the Centers for Disease Control and Prevention. Persistently elevated glucose levels during long-standing diabetes induce structural and functional changes in different proteins in the body, and the replication and the spread of protein aggregates throughout the brain are implicated in the progression of Alzheimer's Disease (A.D.) [2]. For example, the cross-linking of proteins by advanced glycation end products in the vessel wall increases vascular stiffness, and modification of extracellular matrix proteins decreases pericyte adherence. The resulting changes in small vessel structure and function, specifically, expansion of the basement membrane and a loss of vascular cells, may accelerate cognitive decline [3]. Although diabetes can lead to microvascular and macrovascular complications [3], Rouch et al. [4] showed that arterial stiffness is mainly associated with mild cognitive impairment and a higher risk of dementia. Their study on 375 older ambulatory subjects with mild cognitive impairment showed that the conversion to dementia was only associated with increased arterial stiffness and not intima-media thickness, carotid plaques, or carotid artery diameter (after controlling for age and other confounding factors).

Our computational simulations [5] using a detailed multicompartmental neurovascular model provided insights into why vessel stiffness is crucial for hemodynamic oscillatory peaks. In addition to increasing oxygen availability at sites away from small vessels [6], these oscillations support waste clearance within the brain parenchyma, specifically, convective bulk flow drainage along the basement membrane of capillaries and arterial walls [7]. Therefore, changes in the blood vessel pulsatility in type 2 diabetes mellitus (T2DM), including expansion of the basement membrane [3], can impair the convective bulk flow drainage that helps prevent the accumulation of neurotoxic waste proteins, such as protein aggregates, associated with A.D. In a large cohort study [8], younger age at the onset of diabetes increased the risk of subsequent dementia. Interestingly, reduced glucose availability in the CNS can also directly trigger behavioral deficits by promoting amyloid-beta and tau neuropathology and synaptic dysfunction. This reduced glucose availability is related to small vessel dysfunction, reducing oxygen availability at sites distant from small vessels [6]. The question is whether diabetes directly causes early-stage A.D.: these conditions share molecular and cellular features, including insulin resistance and mitochondrial dysfunctions in the brain, such that A.D. has been called "type 3 diabetes" [9].

In this study, we focused on vascular dysfunction in T2DM. Low-frequency Fahræus-Lindqvist-driven (not blood pressure-driven) oscillations in the small vessels are crucial because oscillations in small vessels support nutrient supply [6]. A mechanistic understanding of this is crucial to develop therapeutic measures: vascular factors contribute to cerebrovascular disease and mild cognitive impairment and dementia [10], which are predicted to affect 152 million 
people by 2050 (Alzheimer's Disease International London, UK, 2019). Various physiologically relevant frequency bands have already been identified in the literature: $0.6-2 \mathrm{~Hz}$ and $0.145-0.6 \mathrm{~Hz}$ are related to cardiac and respiratory function, respectively, $0.052-0.145 \mathrm{~Hz}$ is associated with SMC activity, and $0.021-0.052 \mathrm{~Hz}$ may reflect smooth muscle autonomic innervation [11]. Autonomic nerve efferents also regulate metabolic organs: sympathetic nerves increase blood nutrient factors such as glucose by promoting gluconeogenesis, which involves inhibiting insulin secretion, whereas parasympathetic nerves inhibit gluconeogenesis by indirectly promoting insulin secretion [12]. Thus, in the setting of chronic hyperglycemia, any abrupt improvement in glycemic control can also lead to small fiber neuropathy [13] that is postulated to affect the SMCs at pial, penetrating, and precapillary arterioles, where the related oscillatory frequencies are $0.021-0.145 \mathrm{~Hz}$. Then, the low oscillatory frequency $(0.01-0.02 \mathrm{~Hz})$ at capillaries is thought to reflect the Fahræus-Lindqvist effect [6], i.e., the nonlinear dependence of apparent blood viscosity on hematocrit and vessel diameter. Here, blood viscosity and blood glucose are directly related: blood viscosity is lower in prediabetic subjects than in nondiabetic subjects with blood sugar levels that are high but within the normal range [14].

(A)
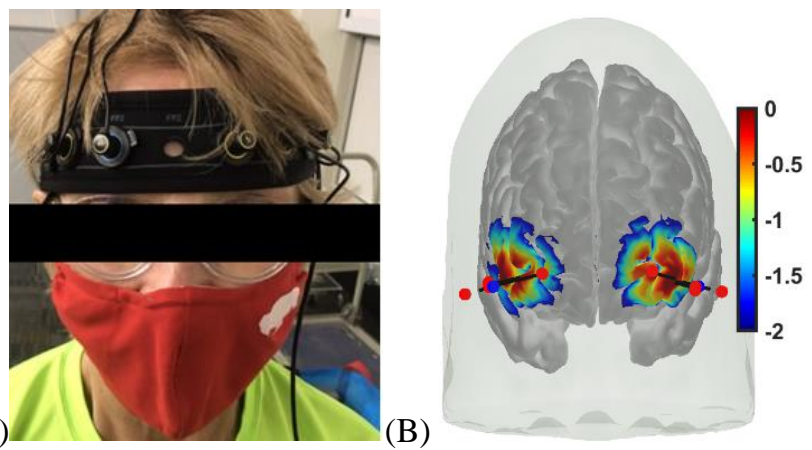

Figure 1. (A) Headband with 4 sources and 2 detectors for fNIRS at the forehead. (B) Sensitivity profile of the optode montage (red dots are sources; blue dots are detectors). The sensitivity values are displayed logarithmically with a default range of 0.01 to 1 , or -2 to 0 in $\log 10$ units.

Cognitive load can significantly reduce blood glucose, such that the amount of cognitive load associated with task performance is an index of its sensitivity to glucose [15]. This is essential because $0.01-0.02 \mathrm{~Hz}$ oscillations support higher oxygen concentrations in areas further from small vessels [6] and may explain the basis of "diabetic brain fog." Therefore, we investigated the relative oscillatory power in the $0.01-$ $0.02-\mathrm{Hz}$ (Fahræus-Lindqvist effect) and $0.021-0.052 \mathrm{~Hz}$ (smooth muscle autonomic innervation) frequency bands using functional near-infrared spectroscopy (fNIRS) of the prefrontal cortex (PFC) during the Mini-Cog assessment for dementia (https://mini-cog.com/) in 60 years and older patients with T2DM and age-matched controls. Moreover, we investigated the PFC activation during memory encoding and recall in Mini-Cog.

\section{METHODS}

\section{A. Experimental setup and subjects}

After written consent, 19 elderly (60 years and older) subjects with T2DM and 38 age-matched controls were recruited. The study was approved by the Institutional Review Board of the University at Buffalo, NY (STUDY00004297). All study procedures were performed according to the local regulations for human subjects' research. Portable brain imaging was performed using four sources and two detectors of a portable fNIRS system (Octamon+, Artinis Medical Systems, Netherlands) at the forehead, as shown in Figure 1A. Figure $1 \mathrm{~B}$ shows the sensitivity profile of the optode montage that covered the middle frontal gyrus (orbital part) and superior frontal gyrus (dorsolateral part) bilaterally [16].
(A)

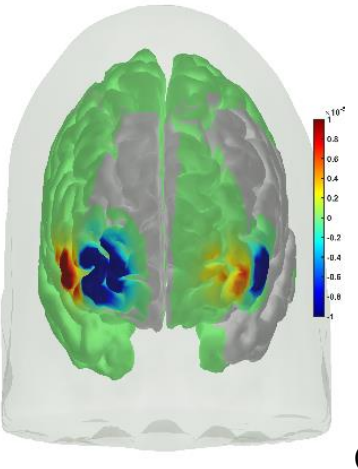

(B)

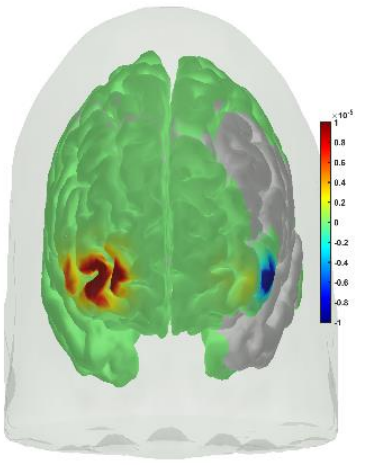

(C)

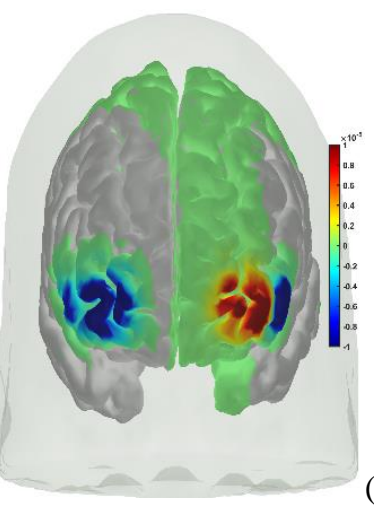

(D)

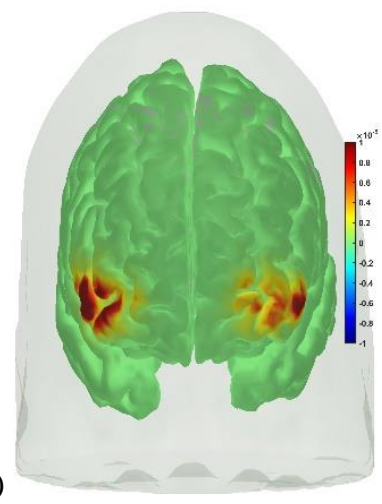

Figure 2: (A) Average cortical activation $(\mathrm{HbO})$ of the prefrontal cortex during memory encoding in 38 age-matched elderly controls. (B) Average cortical activation ( $\mathrm{HbO})$ of the prefrontal cortex during memory recall in 38 age-matched elderly controls. (C) Average cortical activation ( $\mathrm{HbO}$ ) of the prefrontal cortex during memory encoding in 19 elderly subjects with T2DM. (D) Average cortical activation (HbO) of the prefrontal cortex during memory recall in 19 elderly subjects with T2DM.

\section{B. fNIRS data preprocessing}

Preprocessing of the fNIRS data was performed using the standard open-source HOMER3 package (https://github.com/BUNPC/Homer3). The fNIRS preprocessing pipeline followed: first intensity was converted to optical density, and then motion artifacts were detected and filtered with the help of the Savitzky-Golay filtering method 
with default parameters. Then, the optical density was bandpass filtered in the neurovascular coupling band, 0.01$0.1 \mathrm{~Hz}$, then converted to chromophore $(\mathrm{HbO})$ concentration with unit partial pathlength factor. Then, the PCA filter was used to remove the first principal component, then estimate the hemodynamic response function with GLM ('hmrR_GLM') for $-2 \mathrm{sec}$ to $30 \mathrm{sec}$ of the memory encoding and recall parts of the three trials of the Mini-Cog three-item recall test. The cortical activation was estimated using the forward matrix, i.e., the sensitivity profiles (Figure 1B), that provided the transformation from an image of changes in the absorption coefficient in the cortex to the corresponding changes in optical density measured by each source-detector pair [16]. Then, an image of the cortical activation was reconstructed for $5 \mathrm{sec}$ to $30 \mathrm{sec}$ epoch by solving the inverse problem with regularization scaling parameter $=0.01$. Also, the relative power in the $0.01-0.02 \mathrm{~Hz}$ (Fahræus-Lindqvist effect) and $0.021-0.052 \mathrm{~Hz}$ (smooth muscle autonomic innervation) frequency bands were compared between the cohorts using t-test after normality test.

(A)
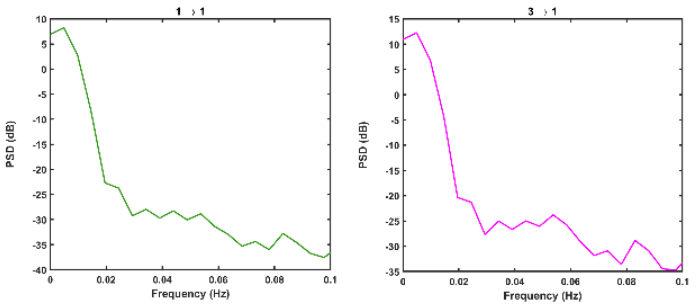

(B)
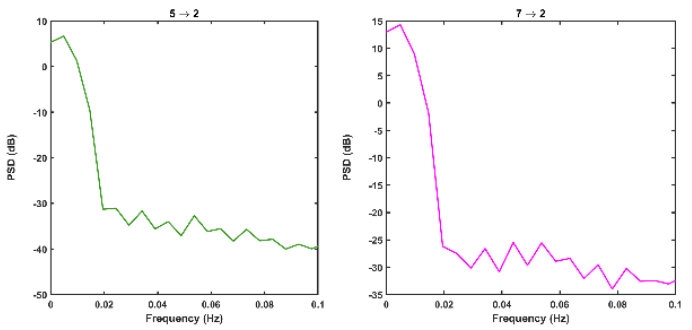

(C)
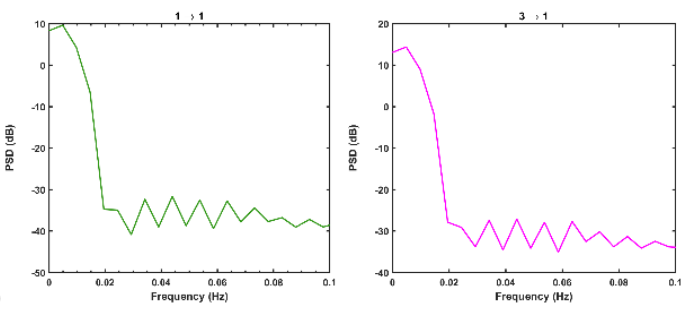

(D)
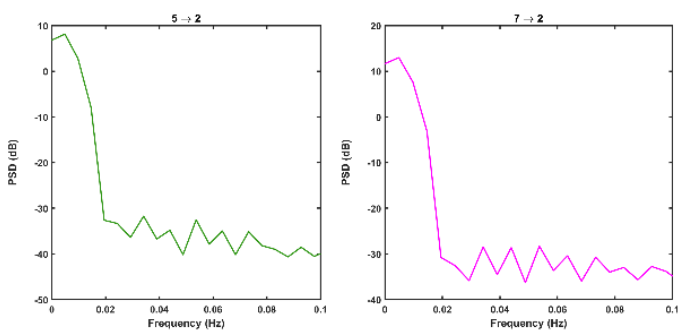

Figure 3: (A) Illustrative power spectral density (PSD) plot of the two fNIRS channels on the left hemisphere in an agematched elderly control. (B) Illustrative PSD plot of the two fNIRS channels on the right hemisphere in an age-matched elderly control. (C) Illustrative PSD plot of the two fNIRS channels on the left hemisphere in an elderly subject with T2DM. (D) Illustrative PSD plot of the two fNIRS channels on the right hemisphere in an elderly subject with T2DM.

\section{RESULTS}

The average cortical activation during memory encoding and recall for the 38 age-matched elderly controls are shown in Figures 2 (A) and 2 (B), respectively. Then, the average cortical activation during memory encoding and recall for the 19 elderly subjects with T2DM are shown in Figures 2 (C) and 2 (D), respectively. During memory encoding, elderly subjects with T2DM had a higher cortical activation at the left dorsolateral PFC than the age-matched elderly controls. Also, during memory encoding, the age-matched elderly controls showed activation of the right middle frontal gyrus (orbital part). During memory recall, the elderly subjects with T2DM had bilateral PFC activation while the age-matched elderly controls showed activation of the right PFC. Therefore, there was greater activation of the left PFC in the elderly subjects with T2DM than the age-matched elderly controls.

Figure 3(A) shows an illustrative power spectral density (PSD) plot of the fNIRS channels on the left hemisphere, whereas 3(B) shows the same for the right hemisphere of an age-matched elderly control. Then, Figure 3(C) shows an illustrative PSD plot of the fNIRS channels on the left hemisphere, whereas 3(D) shows the same for the right hemisphere of an elderly subject with T2DM. Statistical analysis with t-test showed that the age-matched elderly controls had a significantly $(\mathrm{p}<0.01)$ higher relative power in the $0.021-0.052 \mathrm{~Hz}$ (smooth muscle autonomic innervation) frequency band than elderly subjects with T2DM during the Mini-Cog assessment. Moreover, we found a drop in the oscillatory power in the $0.01-0.02-\mathrm{Hz}$ frequency band during the Mini-Cog three-item recall test; this drop was more pronounced in the elderly subjects with T2DM than in the agematched elderly controls.

\section{DISCUSSION}

In this study, we found a difference in the PFC activation between memory encoding and memory recall that has been shown in prior work by Jahani et al. [17]; however, the activation in left dorsolateral PFC during encoding and the activation in dorsolateral PFC bilaterally during recall shown by Jahani et al. was matched our elderly subjects with T2DM better. Moreover, the PSD analysis provided novel insights into the physiology where a significantly lower relative power in $0.021-0.052 \mathrm{~Hz}$ (smooth muscle autonomic innervation) frequency band in elderly subjects with T2DM during the Mini-Cog three-item recall test potentially due to small fiber neuropathy [13] in the pial vessels. Furthermore, a drop in the oscillatory power in the $0.01-0.02-\mathrm{Hz}$ frequency band during Mini-Cog three-item recall test due to cognitive load likely under acute stress [18], where the drop was more pronounced in the elderly subjects with T2DM, possibly because of glucocorticoid receptor signaling in T2DM [19]. Notably, transcranial electrical stimulation induces brain energy consumption that promotes systemic glucose uptake [20]. Specifically, anodal transcranial direct current (tDCS) to induce neuronal excitation causes an energetic depletion 
quantifiable by ${ }^{31} \mathrm{P}$ magnetic resonance spectroscopy. The induced energy consumption promotes systemic glucose tolerance as observed in a standardized euglycemichyperinsulinemic glucose clamp procedure in healthy male volunteers; this tDCS effect may be comparable to the cognitive load-led reduction in blood glucose [15], such that the neurometabolic effects of tDCS evoke regional cerebral blood flow (CBF) [21]. We found that the cerebrovascular reactivity to tDCS following a cerebrovascular accident was significantly less in the lesioned hemisphere, which was postulated to be related to neurovascular coupling [21] and excitation-inhibition status [22]. Therefore, we developed grey-box modeling and causal inference methods to gain a mechanistic understanding of cerebrovascular reactivity to tDCS [5] that captured perivascular modulation at the onset (within $150 \mathrm{~s}$ ) of tDCS. However, longer-term (e.g., $20 \mathrm{~min}$ ) tDCS as a potential treatment for vascular dysfunction in T2DM, which produces a biphasic effect on systemic glucose tolerance [20], needs to be further investigated.

\section{REFERENCES}

[1] T. Vos et al., "Global burden of 369 diseases and injuries in 204 countries and territories, 1990-2019: a systematic analysis for the Global Burden of Disease Study 2019," The Lancet, vol. 396, no. 10258, pp. 1204-1222, Oct. 2020, doi: 10.1016/S01406736(20)30925-9.

[2] G. Meisl et al., "In vivo rate-determining steps of tau seed accumulation in Alzheimer's disease," Science Advances, vol. 7, no. 44, p. eabh1448, doi: 10.1126/sciadv.abh1448.

[3] M. J. Fowler, "Microvascular and Macrovascular Complications of Diabetes," Clinical Diabetes, vol. 26, no. 2, pp. 77-82, Apr. 2008, doi: 10.2337/diaclin.26.2.77.

[4] L. Rouch et al., "Pulse Wave Velocity Is Associated With Greater Risk of Dementia in Mild Cognitive Impairment Patients," Hypertension, 42018-5-2220187-23 2018, Accessed: Sep. 22, 2018.

[5] Y. Arora et al., "Grey-box modeling and hypothesis testing of functional near-infrared spectroscopy-based cerebrovascular reactivity to anodal high-definition tDCS in healthy humans." Sep. 17, 2021. doi: 10.21203/rs.3.rs-83907/v3.

[6] J. B. Geddes, R. T. Carr, F. Wu, Y. Lao, and M. Maher, "Blood flow in microvascular networks: A study in nonlinear biology," Chaos, vol. 20, no. 4, p. 045123, Dec. 2010, doi: 10.1063/1.3530122.

[7] J. Kaur et al., "Waste Clearance in the Brain," Frontiers in Neuroanatomy, vol. 15, p. 53, 2021, doi: 10.3389/fnana.2021.665803.

[8] C. Barbiellini Amidei et al., "Association Between Age at Diabetes Onset and Subsequent Risk of Dementia," JAMA, vol. 325, no. 16, pp. 1640-1649, Apr. 2021, doi: 10.1001/jama.2021.4001.

[9] R. Kandimalla, V. Thirumala, and P. H. Reddy, "Is Alzheimer's disease a Type 3 Diabetes? A critical appraisal," Biochimica et Biophysica Acta (BBA) Molecular Basis of Disease, vol. 1863, no. 5, pp.
1078-1089, May 2017, doi: 10.1016/j.bbadis.2016.08.018.

[10] Y. Pan, H. Li, J. M. Wardlaw, and Y. Wang, "A new dawn of preventing dementia by preventing cerebrovascular diseases," BMJ, vol. 371, p. m3692, Oct. 2020, doi: 10.1136/bmj.m3692.

[11] A. Stefanovska, M. Bracic, and H. D. Kvernmo, "Wavelet analysis of oscillations in the peripheral blood circulation measured by laser Doppler technique," IEEE Trans Biomed Eng, vol. 46, no. 10, pp. 1230-1239, Oct. 1999, doi: 10.1109/10.790500.

[12] J. Imai and H. Katagiri, "Regulation of systemic metabolism by the autonomic nervous system consisting of afferent and efferent innervation," International Immunology, no. dxab023, May 2021.

[13] C. H. Gibbons and R. Freeman, "Treatment-induced neuropathy of diabetes: an acute, iatrogenic complication of diabetes," Brain, vol. 138, no. 1, pp. 43-52, Jan. 2015, doi: 10.1093/brain/awu307.

[14] C. Irace, C. Carallo, F. Scavelli, M. S. De Franceschi, T. Esposito, and A. Gnasso, "Blood viscosity in subjects with normoglycemia and prediabetes," Diabetes Care, vol. 37, no. 2, pp. 488-492, Feb. 2014.

[15] A. B. Scholey, S. Harper, and D. O. Kennedy, "Cognitive demand and blood glucose," Physiol Behav, vol. 73, no. 4, pp. 585-592, Jul. 2001, doi: 10.1016/s0031-9384(01)00476-0.

[16] C. M. Aasted et al., "Anatomical guidance for functional near-infrared spectroscopy: AtlasViewer tutorial," Neurophotonics, vol. 2, no. 2, Apr. 2015, doi: 10.1117/1.NPh.2.2.020801.

[17] S. Jahani et al., "fNIRS can robustly measure brain activity during memory encoding and retrieval in healthy subjects," Scientific Reports, vol. 7, no. 1, Art. no. 1, Aug. 2017, doi: 10.1038/s41598-017-09868-w.

[18] K. Han et al., "Excitation-Inhibition Imbalance Leads to Alteration of Neuronal Coherence and Neurovascular Coupling under Acute Stress," J Neurosci, vol. 40, no. 47, pp. 9148-9162, Nov. 2020.

[19] I. Kokkinopoulou, A. Diakoumi, and P. Moutsatsou, "Glucocorticoid Receptor Signaling in Diabetes," Int J Mol Sci, vol. 22, no. 20, p. 11173, Oct. 2021, doi: 10.3390/ijms222011173.

[20] F. Binkofski et al., "Brain energy consumption induced by electrical stimulation promotes systemic glucose uptake," Biol Psychiatry, vol. 70, no. 7, pp. 690-695, Oct. 2011.

[21] A. Dutta, A. Jacob, S. R. Chowdhury, A. Das, and M. A. Nitsche, "EEG-NIRS based assessment of neurovascular coupling during anodal transcranial direct current stimulation--a stroke case series," J Med Syst, vol. 39, no. 4, p. 205, Apr. 2015.

[22] S. Dagar, S. R. Chowdhury, R. S. Bapi, A. Dutta, and D. Roy, "Near-Infrared Spectroscopy Electroencephalography-Based Brain-StateDependent Electrotherapy: A Computational Approach Based on Excitation-Inhibition Balance Hypothesis," Front Neurol, vol. 7, Aug. 2016. 